\title{
Blood transfusion in veterinary medicine
}

\begin{abstract}
Objective: The Objective of this review article is to discuss and review blood transfusion and its allied practices in dogs, cats, horse, donkey, cattle, sheep and goat including collection, storage and the transfusion. The article discuses new developments, standard practices, protocols and conventional blood product administration techniques.

Conclusion: Blood transfusions is practiced since long back to improve oxygen carrying capacity and rectify the clinical signs of anemia. The potential risks and complications of transfusion may sometimes outweigh the benefits and can cause more harm to the recipient. The improved techniques and facilities for storage of blood have contributed immensely in extending the shelf life of stored blood. Storage of blood also causes storage lesions like erythrocyte survivability. Newer transfusion techniques are being explored such as cell salvage in surgical patients and subsequent autologous transfusion. Xenotransfusions, using blood and blood products between different species, provide an alternative to conventional blood products.
\end{abstract}

Keywords: blood transfusion, autologous, xenotransfusions, veterinary medicine, erythrocytes, hemolytic, antigenic, hemagglutinins, agglutination, diluents, erythropoiesis
Volume 4 Issue 4 - 2017

\author{
Rahul Kumar \\ Department of Veterinary Pathology, College of Veterinary \\ Science \& AH, India \\ Correspondence: Rahul kumar, Department of Veterinary \\ Pathology, College of Veterinary Science \& AH, DUVASU, \\ Mathura, India, Email rahulpoultrypatho@gmail.com
}

Received: February 23, 2017 | Published: May 12, 2017
Abbreviations: NI, neonatal isoerythrolysis; CEA, canine erythrocyte antigen; DEA, dog erythrocyte antigen; ACVM, american college of veterinary internal medicine; PCR, polymerase chain reaction; FeLV, feline leukemia virus; FIV, feline immunodeficiency virus

\section{Introduction}

Blood transfusion is being practiced for centuries for saving life of human beings and animals. Richard Lower in 1665 transfused the blood in a dog for the first time in the history. ${ }^{1}$ With the help of latest techniques and equipment developed after 1950, blood transfusion became more popular in veterinary medicine., ${ }^{2,3}$ Blood transfusion has made considerable advancements in veterinary medicine in recent times. Although, the information and availability of blood and its products has increased, transfusion therapy has become more complex. Advanced screening facilities, blood group testing and techniques for cross matching blood had made the process of donor selection more complicated. Advancement in techniques of separating the components of blood has given the clinician an opportunity to use the component as per the demand of the patient. This article summarizes recent advances in veterinary transfusion medicine and guides the clinicians in decision making while transfusion with evidence.

\section{Blood groups}

Blood groups are named according to the species-specific antigens present on the surface of erythrocytes. Theses antigens play an important role in inducing immune-mediated reactions and can cause complications while transfusing blood from different blood groups. Antigens coupled with platelets, leukocytes and plasma proteins may also induce immune mediated reactions in host animals during transfusion therapies. Plasma also has some naturally occurring all antibodies that can act against other blood groups without any prior exposure to the erythrocyte antigens. Erythrocyte antigens can induce production of antibodies when animals get exposed via blood transfusion, transplacental exposure or in the case of neonatal isoerythrolysis (NI), through colostrum. Blood groups in the common domestic and pet animal species are described here. From a clinician's point of view, these are the antigens to which the veterinary practitioner should be most familiar. However, many other blood group factors and systems have been described and the lack of commercially available typing sera does not diminish the potential significance of these other systems in transfusion medicine.

\section{Dog}

International workshops met in 1972 and 1974 to standardize canine blood groups as defined by isoimmune sera and to standardize canine blood group system nomenclature. ${ }^{4,5}$ The first workshop designated the terminology canine erythrocyte antigen (CEA) followed by a number to indicate the blood group antigen. The second workshop adopted the designation dog erythrocyte antigen (DEA). The new terminology was adopted to avoid confusion with the carcinoembryonic antigen (CEA) system. The blood group system in dogs includes DEA 1.1, DEA 1.2, DEA 3, DEA 4, DEA 5 and DEA 7. The DEA nomenclature system of canine blood group is not accepted worldwide, although some authors use the newer genetic nomenclature system in reporting new blood group specificities. ${ }^{6,7}$ In dogs, naturally occurring alloantibodies are of lesser clinical significance whereas in cats it is very important clinically. ${ }^{89}$ DEA 1.1 and 1.2 are the most important blood groups and are found in $60 \%$ population of canines. ${ }^{9}$ These blood groups can elicit severe transfusion reactions in previously sensitized dogs. DEA 1.3 has been described in German shepherd dogs in Australia. ${ }^{10}$ DEA 4 blood group of dogs is found in high frequency that can cause hemolytic transfusion reactions in DEA 4-negative dogs previously sensitized by DEA 4-positive blood transfusions. ${ }^{11}$ The DEA 3, 5 and 7 blood groups can cause delayed transfusion reactions in dogs lacking these antigens but are previously sensitized to these antigens. ${ }^{12,13}$ 


\section{Cat}

Three blood groups are reported in cats in AB blood group system. Type A blood group is the most common group and found in $95 \%$ of the American cats. ${ }^{8,14}$ Majority of Indian and $30 \%$ of cats in UK belongs to blood group $\mathrm{B} .^{8,15-17}$ blood group $\mathrm{AB}$ is extremely rare but is found in DSH/DLH cats and in breeds in which group B is also found e.g. Abyssinian, Birman, British shorthair, Norwegian forest, Somali, Scottish fold and Persian. ${ }^{18}$ One more erythrocyte antigen, a novel Mik antigen, has been reported in DSH cats. ${ }^{19}$ One should consider the variation in geographical variation in blood groups of felids. The risk of transfusing lethal groups $\mathrm{A}$ or $\mathrm{AB}$ to a cat of blood group B is $20 \%{ }^{17,20}$ Anti-A, anti-B and anti-Mik are naturally occurring alloantibodies found in cats. ${ }^{19,21}$ Alloantibodies that are strong hemagglutinins and hemolysins against type A erythrocytes are very rich in serum of cats with blood group B. Type A cats generally have weak hemagglutinins and hemolysins against type B erythrocytes, hence transfusion reactions are rare. Neonatal isoerythrolysis (NI) occurs in type A or $\mathrm{AB}$ suckling kittens born to type $\mathrm{B}$ queens with transfer of the anti-A alloantibodies via the colostral transfer of immunoglobulin (primarily IgG). ${ }^{14,21,22}$ Transfusion between Mik positive and Mik negative cats can result in acute post-transfusion hemolysins. ${ }^{19}$

\section{Horse and donkey}

The seven blood groups in horses viz. A, C, D, K, P, Q and U are internationally recognized with more than 30 erythrocyte antigens. ${ }^{17,18,23,24}$ Universal donor horse is not possible because of various possible antigenic combinations. The cross matching must be performed although impractical to minimize transfusion reactions. ${ }^{25} \mathrm{Aa}$ and Qa alloantigens are hemolysins and are extremely immunogenic and most cases of NI are associated with anti-Aa or -Qa antibodies. In horses Blood group vary with breeds. Thoroughbreds and Arabian breeds have high prevalence of antigens Aa or Qa whereas, Standard breds lack the Qa antigen. ${ }^{23,26}$ Donkey factor, a unique donkey and mule erythrocyte antigen is not found in the horse and is responsible for neonatal isoerythrolysis in mule pregnancies. ${ }^{27,28}$

\section{Cattle}

The internationally recognized blood groups in cattle are A, B, C, F, J, L, M, R, S, T and Z. out of these 11 groups, group B and J being the most clinically relevant. The $\mathrm{B}$ group itself has more than 60 antigens, thereby making closely matched blood transfusions difficult. The $\mathrm{J}$ antigen is not a true erythrocyte antigen but a lipid found in plasma Cattle having anti-J antibodies with a small amount of adsorbed $\mathrm{J}$ antigen on erythrocytes but negative $\mathrm{J}$ blood group, can develop transfusion reactions when receiving J-positive blood. ${ }^{23,29}$

\section{Sheep and goat}

A, B, C, D, M, R, X are the seven blood groups identified in sheep. The B group has over 52 factors present over erythrocytes. ${ }^{23}$ The $\mathrm{R}$ system in sheep is similar to the $\mathrm{J}$ system in cattle (i.e., antigens are soluble and passively adsorbed to erythrocytes). The M-L blood group in sheep is related to active potassium transport in reticulocytes. ${ }^{30}$ The blood groups of the goat (A, B, C, M, J) are very similar to those of sheep with the B system equally complex. Many of the reagents used for blood typing of sheep also have been used to type goats.

\section{Discussion}

\section{General principles of blood group testing}

Blood group testing can be performed in the clinic to screen potential cat and dog blood donors and to type the recipient for appropriate donor selection prior to crossmatch and transfusion. Commercially available blood typing kits include a card-based agglutination assay, an immunochromatographic cartridge and a gel column diffusion assay (Figure 1). Blood typing cards contain lyophilized antisera in designated reaction wells. The dog cards include positive and negative control wells and the cat cards include an auto control well. A drop of diluent and a drop of whole blood are mixed onto each reaction well on the card, rocked and then observed for macroscopic agglutination. The procedure is simple and results are obtained in less than 2 minutes with no special equipment required. The auto control well included on the cat typing card and on separate cards in dogs allows assessment for auto-agglutination. Auto-agglutination appears similar to a positive reaction and may preclude accurate typing. A prozone phenomenon may occur in the presence of inappropriate antigen: antibody ratios that can generate false negative results. Consequently, an additional drop of diluent should be added to dog samples with weak or grainy reactions followed by rocking and reading the card again and samples from very anemic animals $(\mathrm{PCV}<10 \%)$ should be concentrated prior to blood group testing. ${ }^{31}$

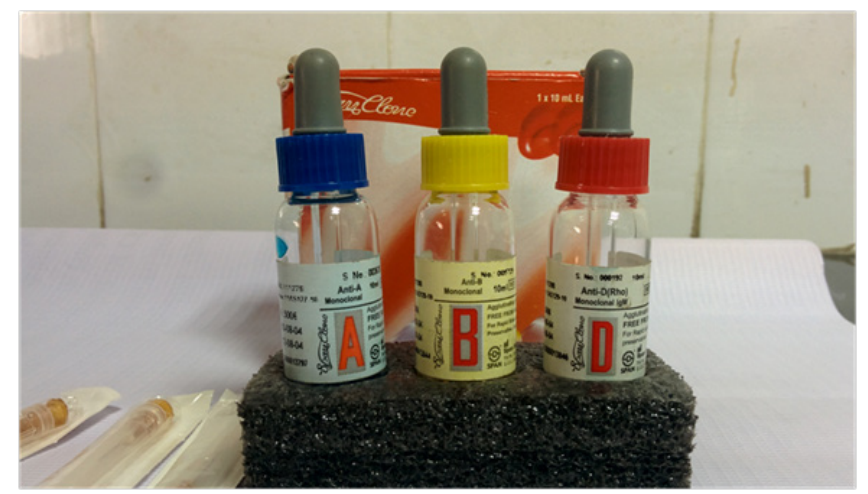

Figure I Kit for testing blood groups (A, B and D blood groups).

The immunochromatographic kits utilize a plastic cartridge device and testing requires about 2 minutes. Testing requires simple preparation of a cell suspension and manipulation of the device to properly place the reaction strip into the suspension. This allows erythrocytes to move up the membrane by capillary action. Erythrocytes positive for the antigen in question are trapped by the antibody impregnated in the strip, which then forms a visible line. Erythrocytes negative for the antigen pass by the antibody and do not form a line. The strip is also impregnated with control material that must read positive to confirm the test was performed properly. These tests are easy to interpret and archive. ${ }^{32}$ The gel tube typing kits require a simple preparation of a cell suspension, a 10-minute incubation period and a 10- minute centrifugation in a centrifuge specifically designed to hold the gel tube cartridges. The reaction is grossly visible as a compact to modestly dispersed layer of agglutinated cells at or near the top of a gel column. Non reacting cells accumulate at the bottom of the column. This method reportedly provides easier result interpretation and higher accuracy for dog and cat typing. ${ }^{33,34}$ Out of all the above mentioned methods of blood group testing, recently developed Gel crossmatch technology is advantageous over the other techniques. One benefit is that the gels can be saved to show to others for verification (vs blood typing cards, which dry up). Gel technology also requires less blood than a standard crossmatch $(0.5 \mathrm{~mL}$ vs $2-3 \mathrm{~mL})$. In addition, the gel crossmatch can be used even if the patient is autoagglutinating. ${ }^{35}$ The gel allows agglutinates to be trapped in a matrix but free cells to sink to the bottom, allowing easier interpretation of compatibility. 


\section{General principles of crossmatching blood}

Major and minor cross matching tests are done for agglutinating and/or hemolytic reactions between donor and recipient. For dog and cat agglutinating tests are sufficient whereas in equines agglutinating and hemolytic tests are required because of presence of agglutinating as well as hemolytic antibodies in equines. ${ }^{36}$ For cattle, sheep and goats test for hemolytic antibodies and complement is necessary. ${ }^{29,37-39}$ The major crossmatch evaluates for the presence (positive findings) or absence (negative findings) of detectable levels of antibodies, whether naturally occurring or induced, in the recipient against donor erythrocyte antigens. A major crossmatch should always be performed in animals that have strong naturally occurring antibodies, as in cats, or in those that may have induced antibodies as from prior transfusions. The latter is true even if the same donor blood is intended for repeated transfusion beyond a span of several days. The minor crossmatch procedure follows the same steps as the major crossmatch but evaluates for the presence or absence of detectable antibodies in donor plasma against recipient erythrocytes. Minor cross matching is of little significance because the volume of plasma donated is very small in comparison to the recipient and is diluted in the recipient particularly in case when only erythrocytes are transfused. ${ }^{31}$ Administration of packed erythrocytes may contain sufficient antibodies against recipient erythrocytes to induce adverse reactions in $\operatorname{dog} s^{40}$ and horses..$^{25}$ An ethylenediaminetetraacetic acid (EDTA) tube and a clot tube from the recipient are preferred for use in crossmatch testing. The EDTA plasma should not be used in place of serum because this contributes to increased rouleaux formation and difficult interpretation of agglutination, particularly in the horse. Preferably, samples should be free of auto-agglutination, hemolysis and lipemia to aid in the interpretation of the reactions. When autoagglutination is present, or when no compatible units are available, transfusing the least incompatible unit may be a necessity, albeit not without significant risk. Test transfusing even a small volume of unmatched blood is an unsafe practice and never recommended. ${ }^{8}$

\section{Crossmatching technique}

Collect the blood from the donor as well as recipient in purple top and red top tubes i.e EDTA tube and nonEDTA tubes respectively. ${ }^{41}$ Centrifuge the blood and allow separating plasma and serum from the RBCs. Remove the serum and save it in a separate sterile tube. Discard the plasma from the EDTA tube. Wash the RBCs collected from EDTA tube. Place the RBCs in a spate tube filled with normal saline and centrifuge for 1 minute. Repeat the process 5 times removing the supernatant every time. Resuspend the cells to make a $2 \%$ to $4 \%$ solution $(0.2 \mathrm{~mL}$ of blood in $4.8 \mathrm{~mL}$ of saline gives a $4 \%$ solution). Label the tubes to make the following mixtures as Major crossmatch (2 drops patient serum with 1 drop donor RBC suspension), Minor crossmatch (1 drop patient RBC suspension with 2 drops donor serum) and Control (1 drop patient RBC suspension with 1 drop patient serum). Incubate the mixtures for 15 to 30 minutes at $37^{\circ} \mathrm{C}$ and then centrifuge for 15 seconds. If either hemolysis or hemagglutination is seen macroscopically, or if agglutination is seen microscopically, the donor is not a good match.

\section{Transfusion therapy: General principles and indications}

Blood transfusion should be practiced after proper blood grouping and cross matching the donor's group with the recipient's to prevent the transfusion reactions. In addition to potential adverse reaction of mismatched blood transfusion, the shortened lifespan of mismatched transfused cells result in ineffective therapy. Breeding females of all the species should be properly checked for bold group and cross matched to dodge primary sensitization and risk of future offspring developing hemolytic disease. In the past, cross matching was recommended in dogs that had previously been pregnant. However, a recent study showed that pregnancy does not seem to sensitize dogs to antigens on RBCs. ${ }^{42}$ Blood grouping for canine DEA 1.1 and for feline types $A$ and $B$ generally practiced in veterinary medicine. ${ }^{33,34}$ Other groups and cross matching can be done in research and reference laboratories. Blood transfusions are mostly risky, hence, they should be performed in only warranted cases. History of previous transfusion therapy should be collected from clients, which necessitates cross matching. Whole blood as well as component is transfused in veterinary medicine depending upon availability and indications of transfusion. The primary indication for blood transfusion is the treatment of severe anemia caused by hemorrhage, hemolysis, ineffective erythropoiesis, immune-mediated hemolytic anemia, chronic inflammatory or infectious disease, or neoplasia. Animals must be clinically evaluated on an individual basis. A thumb rule for the treatment of anemia is to transfuse when the packed cell volume (PCV) is less than $10 \%$ to $15 \% .^{37,43-45}$ Animals with acute-onset anemia, however, usually require transfusion before their $\mathrm{PCV}$ decreases to $15 \%$, which contrasts with the situation in animals with chronic anemia. For cases of thrombocytopenia, the generally accepted trigger for platelet transfusion is platelet counts of $10,000 / \mu \mathrm{L} .{ }^{46}$ Additional indications for transfusion include hypovolemia, primary or secondary clotting factor deficiency and hypoproteinemia. Collected blood should be labeled with all the details and record keeping is crucial in all cases of blood collection and administration.

\section{Selection of donor}

Blood grouping should be performed to select permanent blood donors. All donors should be healthy young adults that have never been transfused. In addition, donors must have undergone routine physical, hematological and clinical chemistry evaluations examinations. Proper clinical history of the expected donor should be collected by carefully interviewing the owner to minimize the risk of disease transmission through blood. In the veterinary medicine, it is usually the cost that restricts to test individual units. Therefore, a combination of careful interview and blood screening of the donor is used to minimize the risk of infectious disease transmission. Donor should be properly vaccinated and should be tested free of blood parasites and other infectious diseases. In 2005, the American College of Veterinary Internal Medicine (ACVIM) Consensus Statement on infectious disease testing for blood donors was published. ${ }^{47}$ Since publication, polymerase chain reaction (PCR) assays have become more readily available Several veterinary diagnostic laboratories offer donorscreening PCR panels, which typically include at least Ehrlichia spp, Babesia spp, Anaplasma spp and Mycoplasma hemocanis or Mycoplasma haemofelis. For many diseases of concern. ${ }^{3}$ Donors should have normal baseline PCV and total protein concentrations prior to any donation. Blood should be collected aseptically usually via jugular venipuncture. To avoid interference with platelet function, donors should not be sedated with acepromazin. ${ }^{48}$

\section{Dogs}

$15 \mathrm{ml}$ of blood per $\mathrm{kg} \mathrm{BW}$ can be collected from dog in every 6 weeks. ${ }^{13}$ Dogs with a history of previous blood transfusion should not be used as donors. ${ }^{8}$ Dogs negative for DEA 1.1 can be considered universal donors for first-time transfusion recipients. ${ }^{8} \mathrm{~A}$ dog is considered a universal donor when negative for DEA 1.1, 1.2, 3, 5, 7 and positive for DEA $4 .{ }^{9,13}$ Use of universal donors is recommended 
to minimize potential sensitization of the recipient and improve the odds of identifying compatible donors when periodic transfusions are expected. Approximately 50\% dogs are positive for DEA 1.1 and transfusion between DEA 1.1 positive donor and recipient is sensible. ${ }^{8,9}$ Practically DEA 1.1 negative dogs are ideal for first time transfusions regardless of recipient blood type and DEA 1.1 positive donors should be limited to DEA 1.1 positive recipients. Dogs to be used as donors should be heavier than $25-30 \mathrm{~kg}$, bled less than once in four weeks to prevent iron deficiency and well nourished including oral iron supplementation. To ensure general good health donors should be checked for fecal and heartworm disease. According to the American College of Veterinary Internal Medicine's (ACVIM) agreement, donors should be test negative for transmissible infectious diseases like babesiosis, leishmaniasis brucellosis, ehrlichiosis, anaplasmosis, trypanosomiasis, bartonellosis and hemoplasmosis and neorickettsiosis. $^{47}$

\section{Cats}

10 and $12 \mathrm{~mL}$ of blood $/ \mathrm{kg}$ body weight can be collected for transfusion from donor. Healthy adult cats can donate $45-60 \mathrm{~mL}$ every 6weeks. ${ }^{49,50}$ Cats need to be sedated for blood collection. Like dogs, donors should have negative fecal and heartworm disease examinations as part of a general health checkup. Donor cat should be checked for blood group before selection. There is no universal cat donor because of the cat's naturally occurring alloantibodies. Donor cats should be negative for feline leukemia virus (FeLV), feline immunodeficiency virus (FIV), cytauxzoonosis, anaplasmosis, bartonellosis, ehrlichiosis and neorickettsiosis and hemoplasmosis. ${ }^{47}$

\section{Horses}

Adult horses can safely donate approximately $6-8 \mathrm{~L}$ of blood. Whole blood can be collected every 15-30days and plasma collected every 7 days if the erythrocytes are returned to the donor. ${ }^{51}$ Donor should be healthy, negative for equine infectious anemia and should have normal PCV and plasma protein concentrations. Male donors are preferred as they are less likely to have been previously sensitized. ${ }^{52}$ Mares that have been pregnant or foaled and horses that have received blood or erythrocyte-contaminated plasma transfusions should be excluded as potential donors. A totally compatible blood transfusion is practically not possible in horses. Cross matching to identify the most compatible donor is recommended to avoid adverse transfusion reactions but it is not possible to identify all donor/ recipient incompatibilities. ${ }^{25}$ Because Aa and Qa erythrocyte antigens are extremely immunogenic, Aa- and Qa-negative donors are the best choice as donors to recipients of unknown blood type. In cases of NI, the dam's washed erythrocytes may be used for transfusion to severely anemic foals whereas a transfusion from the sire to foal is contraindicated. ${ }^{23,52}$ Mule foals with NI could receive a transfusion from a horse not previously sensitized by pregnancy against donkey factor because horses are known to be free of naturally-occurring antibodies against donkey factor.

\section{Cattle and sheep}

Cattle can donate $8-14 \mathrm{~mL}$ of blood per $\mathrm{kg}$ of body weight Perfectly or closely crossmatch blood transfusions are very difficult in cattle. First transfusions are generally of low risk, but ideally a donor would be negative for the $\mathrm{J}$ antigen. ${ }^{48}$ Cases of transmission of Prion diseases by blood transfusion have been reported in sheep ${ }^{47}$ therefore, screening should be done for prion disease prior to blood transfusion in ruminants.

\section{Anticoagulants used for storage}

Two anticoagulants namely Citrate-phosphate-dextrose-adenine (CPDA-1) and Acid-citrate-dextrose (ACD) are commonly used for storage of blood. CPDA-1 is considered better anticoagulant because it maintains higher levels of 2,3-disphosphoglycerate (2,3-DPG) and adenosine triphosphate (ATP) in collected blood. In CPDA-1 blood can be stored for approximately 35days. Acid-citrate-dextrose (ACD) allows storage of blood for 21 days..$^{13,49,53,38}$ Use $1 \mathrm{~mL}$ of anticoagulant (CPDA-1/ACD) for every $7 \mathrm{~mL}$ of blood. Blood should be refrigerated in plastic blood collection bags. Heparin is not recommended for blood collection because it activates platelets, but if still used $5 \mathrm{U}$ per $\mathrm{mL}$ of blood is sufficient. 6 Blood collected in heparin as anticoagulant must be used immediately. Survival and functional usefulness of erythrocytes decrease with increased storage temperature and time because of glucose consumption and depletion of ATP and 2,3DPG. Blood should be collected into latex free plastic bags or plastic syringes to preserve platelets. ${ }^{54}$

\section{Transfusion process}

Aseptic conditions should be maintained and perfect aseptic procedures should be followed while collection of blood for transfusion. According to ACVIM, a separate aliquot from every donated unit of blood should be stored for later testing when disease transmission following transfusion is suspected.$^{47}$ Blood should be filtered using $150-170 \mu \mathrm{m}$ pore nonlatex filters either prior to or during administration. Blood should be warmed to $37^{\circ} \mathrm{C}$ before administration to prevent hypothermia. Temperature should not be more than $37^{\circ} \mathrm{C}$, because higher temperatures cause lysis of erythrocytes and inactivation of clotting factors. Blood is administered intravenously through commercially available administration I/V sets with filters. Fluid containing $0.9 \%$ saline should be used when concurrent crystalloid fluid therapy is indicated or for reconstituting blood components such as packed erythrocytes. Lactated Ringer's solution causes calcium chelation with citrate-containing anticoagulants and subsequent clot formation, $5 \%$ dextrose in water cause swelling and lysis of erythrocytes and hypotonic saline fluids will lyse erythrocytes, so they are contraindicated.

Excessive and rapid injection of blood or plasma can result in circulatory overload and heart failure. Generally, blood should be given intravenously at a rate not exceeding $10 \mathrm{~mL} / \mathrm{kg}$ per hour (always begin the transfusion slowly then gradually increase flow rate); however, each patient must be assessed individually to establish an appropriate infusion rate. For example, hypovolemic patients may require an infusion rate of $20 \mathrm{~mL} / \mathrm{kg}$ per hour, whereas patients with cardiac, renal, or hepatic disease or recumbent calves may require an infusion rate of only $1 \mathrm{~mL} / \mathrm{kg}$ per hour. ${ }^{13,48}$ If blood is transfused too quickly, salivation, vomiting and muscle fasciculations may occur. Warm blood should be transfused within 4 hours to avoid contamination. The volume of blood to be transfused is determined according to the recipient's body weight, estimated blood volume, PCV of the recipient and of the donor and the purpose of therapy. A simple guideline for small animals is that $10-15 \mathrm{~mL} / \mathrm{kg}$ of packed erythrocytes or $20 \mathrm{~mL} / \mathrm{kg}$ of whole blood increases the PCV by $10 \%$ if the donor has a PCV of approximately $40 \% .{ }^{13,55}$ One report in horses demonstrated that $15 \mathrm{~mL} /$ $\mathrm{kg}$ of whole blood and $8-10 \mathrm{~mL} / \mathrm{kg}$ of packed erythrocytes resulted in a $4 \%$ increase in PCV when the donor PCV was $35-40 \%{ }^{25}$ More specific calculations for cattle are reported depending on the indication for transfusion for example, in hemorrhagic shock a general volume rule is $7 \mathrm{~L}$ of whole blood $/ 600 \mathrm{~kg}$ cow. ${ }^{21}$ For cases of thrombocytopenia 
in dogs and cats for which fresh whole blood is used for treatment, the general rule is to administer $10 \mathrm{~mL} / \mathrm{kg}$ to increase the recipient's platelet count by a maximum of about $10,000 / \mu \mathrm{L} .{ }^{46}$ In dogs, the half-life of erythrocytes transfused after matching is approximately 21 days. In cats, the half-life of erythrocytes transfused after matching is approximately $30-38$ days. ${ }^{56}$ In horses and cattle the survival time of compatible transfused erythrocytes is only 2 -4days. ${ }^{57,58}$

Preparations while transfusion: Fresh whole blood is indicated for transfusion in case of acute hemorrhage, anemia, coagulation disorders and thrombocytopenia. Stored whole blood is indicated for transfusion in anemia but will not provide platelets or coagulation factors. Packed erythrocytes are recommended for anemic animals, particularly those at high risk for volume overload. Fresh-frozen or stored-frozen plasma is used in cases of congenital or acquired deficiencies of coagulation factors and hypoproteinemia. Fresh-frozen plasma is indicated for use in failure of passive transfer (hypogammaglobulinemia) in calves, foals, puppies and kittens. ${ }^{37,38,59,60}$ Platelet-richplasma is indicated for severe thrombocytopenia or thrombocytopathia. Hyperimmune equine plasma or equine plasma rich in anti-endotoxin antibodies has been used for critically ill foals recovering from septicemia. ${ }^{6}$ Hyperimmune serum products are also available for use in cattle with infectious disease. ${ }^{57}$ Blood substitutes are available and have been used for treatment of anemia in different species of animals including dogs, cats, horses, birds and ferrets. Few advantages of blood substitutes include no requirement of blood grouping and cross matching, risk of infectious disease transmission is minimized and the shelf life is long. However, the product is expensive and must be discarded if not used within 24 hours. It has a half-life of 18-40hours, depending on the dosage once administered. One should be aware of the potential for abuse of artificial oxygen-carriers in canine and equine athletes. ${ }^{59}$ Lastly, these products can interfere with patient monitoring using colorimetric laboratory tests. The effect of transfusion of blood substitutes in recipients should be monitored using hemoglobin concentration, not PCV. ${ }^{13,59}$

Transfusion reactions and sequelae: Potential transfusion reactions may be acute or delayed. Incompatible transfusions may cause acute intravascular hemolysis leading to hemoglobinemia and hemoglobinuria. Release of thromboplastic substances may lead to disseminated intravascular coagulopathy. Mismatch transfusion may cause release of vasoactive amine leading to hypotension, shock, acute renal failure and death. Delayed hemolysis is evidenced by a decrease in PCV between 2 to 14days after transfusion. It is most common in previously transfused animals with an antibody titer too low to detect by cross matching. Generally, extravascular hemolysis causes hyperbilirubinemia and bilirubinuria. If the recipient has not been previously transfused then the first transfusion is usually safe regardless of the donor's blood group, because alloantibodies against the common canine erythrocyte antigens 1.1 and 1.2 do not exist and sensitization does not occur during pregnancy in dogs. Administering a mismatched first transfusion may sensitize the recipient to immunogenic antigens such as 1.1, 1.2, 7 and others, however and result in shortened survival times of the transfused cells on first transfusion and subsequent predisposition to severe transfusion reaction. The strongest antigen in dogs, DEA 1.1, elicits the most severe transfusion reaction. ${ }^{9} \mathrm{AB}$-mismatched transfusions of any phase in cats may cause acute hemolytic incompatibility reactions. Erythrocytes are destroyed immediately in cats because of alloantibodies in contrast to the delayed transfusion reactions in dogs. Transfusion of type B blood to a type A cat cause shortened erythrocyte survival, thus resulting in ineffective therapy. Even in the first transfusion of type A blood to a type B cat results in an acute hemolytic transfusion reaction with massive intravascular hemolysis leading to serious clinical signs and possibly death. ${ }^{14,22}$ Cats with $\mathrm{AB}$ blood group can safely receive type AB or A blood. Transfusion of Mik-positive blood to Mik-negative recipients cats can also result in acute hemolytic transfusion reactions. ${ }^{19}$ After identification of novel blood groups in cats, even $\mathrm{AB}$-matched transfusions can result in reactions, thus, cross matching is mandatory for blood or plasma transfusion in felids. ${ }^{8}$ First transfusions in cattle are of low risk, whereas transfusing J-positive erythrocytes to J-negative cattle recipient can result in transfusion reactions. In J-antigen mismatched second transfusion within four days of first transfusion can result in hemolytic reactions..$^{37}$ Neonatal isoerythrolysis is the destruction of erythrocytes in the circulation of offspring by alloantibodies of maternal origin that are absorbed from colostrum. Kittens at risk include those that are type $\mathrm{A}$ or $\mathrm{AB}$ that suckle colostrum from type $\mathrm{B}$ queens in the first 16 hours of life. ${ }^{8}$ Nearly all cases of NI in foals are caused by factor $\mathrm{Aa}$ in the A system and factor Qa in the Q system.23 Signs of transfusion reactions usually develop 24-36hours after suckling like anemia, liver failure and kernicterus (bilirubin encephalopathy) being the primary causes of death in foals. ${ }^{62}$ Complications other than erythrocyte antigen-antibody reactions include fever, allergic reactions, circulatory overload, citrate toxicosis, ammonia toxicosis and infection. ${ }^{13,63-65}$ Fever is a common clinical manifestation of blood transfusion reaction. It may occur in response to leukocyte or platelet antigens or because of sepsis from bacterial contamination of blood. Sensitivity to plasma proteins or leukocyte and platelet antigens is generally responsible for allergic reactions after transfusions in dogs, cattle and horses. ${ }^{13,25,37}$ Circulatory overload is a potential sequele when whole blood is transfused in patients with compromised cardiac function. Citrate toxicity can be serious in hypocalcaemic cattle. ${ }^{35}$ Prolonged storage of blood can cause ammonia toxicity. Patients with liver diseases should be closely monitored for such a case. Blood borne parasites and viruses should be considered specially and contamination of blood in case of prolonged storage or slow transfusion should be kept in mind by the veterinary practitioner. The health checkup and screening of donors should be strictly practiced and to minimize the risk due care of the collected blood while storing should be taken of prior to transfusion. ${ }^{66}$ The blood unit collected from the donor should be properly labeled and an aliquot should be retained for testing is needed. Cases of serum hepatitis, liver failure and kernicterus have been reported in equines transfused with commercially available plasma. ${ }^{47,67}$ Visible hemolysis of the donor unit suggests inappropriate storage or bacterial contamination, in which case culture is warranted. Blood samples from the donor as well as recipient before and after transfusion should be collected for confirmation of blood group, cross matching and status of Coomb's test. ${ }^{65}$

\section{Conclusion}

Transfusion medicine may be a life saving modality in case of emergency or critically ill animals. Blood products are becoming readily available and transfusions can be performed in many veterinary clinics. The appropriate use of transfusion medicine should balance the rare but not negligible potential risks associated with transfusions. Patients should be appropriately screened with blood typing and cross matching before transfusion. Ongoing research may provide even better platform typing, longer storage times and a larger variety of products that are more specific for each species. 


\section{Acknowledgements}

None.

\section{Conflict of interest}

The author declares no conflict of interest

\section{References}

1. Lower R. The success of the experiment of transfusing the bloud of one animal into another. Philos Trans R Soc Lond B Biol Sci. 1665;1:352.

2. Cotter SM. History of transfusion medicine. Adv Vet Sci Comp Med. 1991;36:1-8.

3. Davidow B. Transfusion medicine in small animals. Vet Clin Small Anim. 2013;43(4):735-756.

4. Vriesendorp HM, Albert ED, Templeton JW, et al. Joint report of the second international workshop on canine immunogenetics. Transplant Proc. 1976;8(2):289-314.

5. Vriesendorp HM, Westbroek DL, D'Amaro J, et al. Joint report of $1^{\text {st }}$ international workshop on canine immunogenetics. Tissue Antigen. 1973;3(2):145-163.

6. Symons M, Bell K. Expansion of the canine A blood group system. Anim Genet. 1991;22(3):227-235.

7. Symons M, Bell K. Canine blood groups: description of 20 specifi cities. Anim Genet. 1992;23:509-515.

8. Giger U. Blood-typing and crossmatching. In: Bonagura JD, et al. editors. Kirk's Current Veterinary Therapy XIV. 1st ed. St. Louis, USA: Saunders Elsevier; 2009. p. 260-265.

9. Hohenhaus A. Importance of blood groups and blood group antibodies in companion animals. Transfus Med Rev. 2004;18(2):117-126.

10. Symons M, Bell K. Expansion of the canine A blood group system. Anim Genet. 1991;22(3):227-235.

11. Melzer K, Wardrop K, Hale A, et al. A hemolytic transfusion reaction due to DEA 4 alloantibodies in a dog. J Vet Intern Med. 2003;17(6):931-933.

12. Hale A. Canine blood groups and their importance in veterinary transfusion medicine. Vet Clin North Am Small Anim Pract. 1995;25(6):1323-1332.

13. Wardrop K. Transfusion medicine. In: Morgan RV editors. Handbook of Small Animal Practice. 5th ed. St. Louis, USA: Saunders Elsevier; 2008. p. 707-713.

14. Giger U, Bucheler J, Patterson D. Frequency and inheritance of A and B blood types in feline breeds of the United States. J Hered. 1991;82(1):1520.

15. Giger U, Griot-Wenk M, Bucheler J, et al. Geographical variation of the feline blood type frequencies in the United States. Fel Pract. 1991;19:21-26.

16. Malik R, Griffin D, White J, et al. Prevalence of the feline A/B blood type in the Sydney region. Aust Vet J. 2005;83(1-2):38-44.

17. Forcada Y, Guitian J, Gibson G. Frequencies of feline blood types at a referral hospital in the South East of England. J Small Anim Pract. 2007:48(10):570-573.

18. Wenk MG, Callan M, Casal M, et al. Blood type AB in the feline AB blood group system. Am J Vet Res. 1996;57(10):1438-1442.

19. Weinstein N, Blais M, Harris K, et al. A newly recognized blood group in domestic shorthair cats: the Mik red cell antigen. $J$ Vet Intern Med. 2007;21(2):287-292.
20. Knottenbelt C, Addie D, Day M, et al. Determination of the prevalence of feline blood types in the UK. J Small Anim Pract. 1999;40(3):115-118.

21. Bucheler J, Giger U. Alloantibodies against A and B blood types in cats. Vet Immunol Immunopathol. 1993;38(3-4):283-295.

22. Giger $U$. The feline $A B$ blood group system and incompatibility reactions. In: Kirk RW, et al. editors. Current Veterinary Therapy XI. Philadelphia, USA; 1992. p. 470-474.

23. Blackmer J, Parish S. Diseases caused by allogeneic incompatibilities In: Smith BP editor. Large Animal Internal Medicine. 3rd ed. St. Louis, USA: Mosby Elsevier Science; 2002. p. 1604-1613.

24. Stormont C, Suzuki Y, Rhode E. Serology of horse blood groups. Cornell Vet. 1964;54:439-452.

25. Hurcombe S, Mudge M, Hinchcliff K. Clinical and clinicopathologic variables in adult horses receiving blood transfusions: 31 cases (19992005). J Am Vet Med Assoc. 2007;231(2):267-274.

26. Wilkins P. Disorders of foals. In: Reed SM, et al. editors. Equine Internal Medicine. 2nd ed. St. Louis, USA: Saunders Elsevier; 2004. p. 14021431.

27. Mc Clure J, Koch C, Traub-Dargatz J. Characterization of a red blood cell antigen in donkeys and mules associated with neonatal isoerythrolysis. Anim Genet. 1994;25(2):119-120.

28. Traub-Dargatz J, McClure J, Koch C, et al. Neonatal isoerythrolysis in mule foals. J Am Vet Med Assoc. 1995;206(1):67-70.

29. Stormont C. Blood groups in animals. Adv Vet Sci Comp Med. 1991;36:955 .

30. Tucker EM, Ellory JC. The M-L blood group system and active potassium transport in sheep reticulocytes. Anim Blood Groups Biochem Genet. 1971;2(2):77-87.

31. Giger U. Blood typing and crossmatching to ensure compatible transfusions. In: Bonagura JD editor. Kirk's Current Veterinary Therapy XIII. Philadelphia: Philadelphia, USA: WB Saunders; 2000. p. 396-399.

32. Seth M, Winzelberg S, Jackson K, et al. Comparison of gel column, card and cartridge techniques for DEA 1.1 blood typing of dogs. J Vet Intern Med. 2008;22:775.

33. Giger U, Stieger K, Palos H. Comparison of various canine blood-typing methods. Am J Vet Res. 2005;66(8):1386-1392.

34. Stieger K, Palos H, Giger U. Comparison of various bloodtyping methods for the feline AB blood group system. Am J Vet Res. 2005;66(8):13931399.

35. Novaretti MC, Jens E, Pagliarini T, et al. Comparison of conventional tube test technique and gel microcolumn assay for direct antiglobulin test: a large study. J Clin Lab Anal. 2004;18(5):255-258.

36. Jain N. Hematologic techniques. In: Jain NC editor. Schalm's Veterinary Hematology. 4th ed. Philadelphia, USA; 1986. p. 71-74.

37. Divers T. Blood component transfusions. Vet Clin North Am Food Anim Pract. 2005;21:615-622.

38. Jain N. Immunohematology. In: Jain NC editor. Essentials of Veterinary Hematology. Philadelphia, USA; 1993. p. 381-407.

39. Stormont C. Blood groups in animals. J Am Vet Med Assoc. 1982;181:1120-1124.

40. Kerl MK, Hohenhaus A. Packed red blood cell transfusions in dogs: 131 cases (1989). J Am Vet Med Assoc. 1993;202(9):1495-1499.

41. Lanevschi A, Wardrop KJ. Principles of transfusion medicine in small animals. Can Vet J. 2001;42(6):447-454. 
42. Blais MC, Berman L, Oakley DA, et al. Canine Dal blood type: a red cell antigen lacking in some Dalmatians. $J$ Vet Intern Med. 2007;21(2):281286.

43. Kristensen A, Feldman B. Blood banking and transfusion medicine. In: Ettinger SJ, et al. editors. Textbook of Veterinary Internal Medicine. 4th ed. Philadelphia, USA: WB Saunders; 1995. p. 347-360.

44. Mitchell E, Johns J. Avian hematology and related disorders. Vet Clin Exot Anim. 2008;11(3):501-522.

45. Sellon D. Disorders of the hematopoietic system. In: Reed SM editor Equine Internal Medicine. 2nd ed. St. Louis, USA: Saunders Elsevier; 2004. p. 721-768

46. Ogg AA. Triggers for prophylactic use of platelet transfusions and optimal platelet dosing in thrombocytopenic dogs and cats. Vet Clin North Am Small Anim Pract. 2003;33(6):1401-1418.

47. Wardrop KJ, Reine N, Birkenheuer A, et al. Canine and feline blood donor screening for infectious disease. J Vet Intern Med. 2005;19(1):135-142.

48. Hackett TB, Jensen WA, Lehman TL, et al. Prevalence of DNA of Mycoplasma haemofelis, Candidatus Mycoplasma haemominutum, Anaplasma phagocytophilum and species of Bartonella, Neorickettsia and Ehrlichia in cats used as blood donors in the United States. J Am Vet Med Assoc. 2006;229(5):700-705.

49. Lucas R, Lentz K, Hale A. Collection and preparation of blood products Clin Tech Small Anim Pract. 2004;19(2):55-62.

50. Roux F, Deschamps J, Blais M, et al. Multiple red cell transfusions in 27 cats (2003-2006): indications, complications and outcomes. J Fel Med and Surg. 2008;10(3):213-218.

51. Byars T, Divers T. Clinical use of blood transfusions. Calif Vet 1981;1:14-16.

52. Durham AE. Blood and plasma transfusion in the horse. Equine Vet Educ. 1996;8(1):8-12.

53. Bucheler J, Cotter S. Storage of feline and canine whole blood in CPDA1 and determination of post-transfusion viability. $J$ Vet Intern Med. 1994;8:172.
54. Mudge MC, MacDonald M, Owens S, et al. Comparison of 4 blood storage methods in a protocol for equine preoperative autologous donation. Vet Surg. 2004;33(5):475-486.

55. Lichtenberger M. Transfusion medicine in exotic pets. Clin Tech Small Anim Pract. 2004;19(2):88-95.

56. Marion R, Smith J. Survival of erythrocytes after autologous and allogeneic transfusion in cats. J Am Vet Med Assoc. 1983;183(12):14371439 .

57. Kallfelz F, Whitlock R. Survival of 59Fe-labeled erythrocytes in crosstransfused bovine blood. Am J Vet Res. 1973;34(8):1041-1014.

58. Kallfelz F, Whitlock R, Schultz R. Survival of 59 Fe-labeled erythrocytes in cross-transfused equine blood. Am J Vet Res. 1978;39(4):617-620.

59. Chiaramonte D. Blood-component therapy: selection, administration and monitoring. Clin Tech Small Anim Pract. 2004;19(2):63-67.

60. Crisman M, Scarratt W. Immunodeficiency disorders in horses. Vet Clin Equine. 2008;24(2):299-310.

61. Peek S, Semrad S, McGuirk S, et al. Prognostic value of clinicopathologic variables obtained at admission and effect of antiendotoxin plasma on survival in septic and critically ill foals. J Vet Intern Med. 2006;20(3):569574

62. Polkes AC, Giguere S, Lester G, et al. Factors associated with outcome in foals with neonatal isoerythrolysis (72 Cases, 1998-2003). J Vet Intern Med. 2008;22(5):1216-1222.

63. Harrell K, Kristensen A, Parrow J. Canine transfusion reactions. Part I. Causes and consequences. Compend Cont Educ. 1997;19:181-190.

64. Harrell K, Kristensen A, Parrow J. Canine transfusion reactions. Part II Prevention and treatment. Compend Cont Educ. 1997;19:193-200.

65. Hohenhaus A. Transfusion reactions. In: Feldman BF, et al. editors Schalm's Veterinary Hematology. 5th ed. Lippincott, Philadelphia, USA Williams \& Wilkins; 2000. p. 864-868.

66. Wardrop K, Reine N, Birkenheuer A, et al. Canine and feline blood donor screening for infectious disease. J Vet Intern Med. 2005;19:135-142. 